\title{
LA ENSEÑANZA DE LAS PRIMERAS LETRAS EN EL FERROL DE FINALES DEL ANTIGUO RÉGIMEN
}

\author{
Alfredo MARTíN GARCÍA \\ Universidad de León
}

\begin{abstract}
RESUMEN: El Ferrol de la segunda mitad del siglo XVIII no sólo experimentó una intensa transformación demográfica, social o económica, también vivió cambios trascendentales en el campo de la educación y la cultura. Nuestro objetivo en este trabajo es analizar el mundo de la enseñanza de las primeras letras, calibrando sus repercusiones en la sociedad ferrolana y realizando la necesaria comparación con el panorama urbano gallego y español
\end{abstract}

PALABRAS CLAVE: Ferrol, Galicia, educación, siglo XVIII.

ABSTRACT: In the second half of 18th century, the city of Ferrol not only shows a important demographic, social and economic transformations but also show a significant change in the world of education and culture. Our objective in this work is to analyse the characteristics of the elementary education in Ferrol. We will measure its repercussions in the society of the city and we will compare this example with other Spanish cases.

KEYWORDS: Ferrol, Galicia, education, $18^{\text {th }}$ century.

\section{INTRODUCCIÓN}

La real villa de Ferrol nace a mediados del siglo XVIII como verdadero centro urbano, a raíz de la ubicación en su rada del grandioso arsenal-astillero de la Corona ${ }^{1}$. Los cambios que esa decisión política generaron en su configuración demográfica, económica o social han sido analizados en profundidad en estos

${ }^{1}$ El presente trabajo forma parte del proyecto "Comunicación y difusión en la Galicia del Antiguo Régimen: cultura oral y cultura escrita en una sociedad bilingüe”, financiado por el Ministerio de Educación y Ciencia (Ref. HUM2005-01289). 
últimos años ${ }^{2}$. Sin embargo, muy poco es lo que se sabe de las transformaciones que pudo provocar en el campo de la cultura y la educación ${ }^{3}$.

Durante la segunda mitad del aquella centuria, convivieron en la localidad centros educativos de marcado sabor tradicional -las llamadas "escuelas de primeras letras" o la cátedra de gramática-, con otros de alto nivel científico, estrechamente vinculados al reformismo borbónico, caso, por ejemplo, de la academia de guardiamarinas. Como un primer paso para un estudio más ambicioso del mundo de la educación en Ferrol, nos proponemos en este trabajo analizar la base de aquel engranaje en el siglo XVIII, esto es, la enseñanza primaria. El conocimiento de ese primer estadio del sistema educativo nos ha de servir como trampolín para posteriores trabajos.

\section{LA ENSEÑANZA DE LAS PRIMERAS LETRAS EN LA ESPAÑA DEL SIGLO XVIII}

Siguiendo un criterio optimista en exceso, prácticamente todos los tratadistas españoles del siglo XVIII, desde Jovellanos y Olavide a Picornell o Campomanes, pasando por Manuel de Aguirre, entre otros, relacionaban estrechamente la felicidad de los pueblos con la instrucción. En opinión de estos ilustrados, sin el decisivo concurso de la educación, no podía existir de ningún modo felicidad, tanto a nivel individual como colectivo. Evidentemente, cuando empleaban el término "felicidad", no lo enfocaban desde un punto de vista exclusivamente anímico sino, más bien, desde la premisa que afirmaba que una sociedad era más feliz cuanto más riqueza poseía ${ }^{4}$. Esos mismos criterios, tomados por los pensadores españoles de Locke y los ilustrados franceses ${ }^{5}$, también imperaban dentro de la minoría culta del Ferrol dieciochesco. Pero junto a esa reflexión un tanto idealista, existían razones mucho más pragmáticas que vinculaban la educación con la

2 Martín García, A. (2003). Una sociedad en cambio. Ferrol a finales del Antiguo Régimen, Ferrol: Embora 2003; MARTín GARCíA, A. (2005). Demografía y comportamientos demográficos en la Galicia de la Edad Moderna. La Real Villa de Ferrol y su Tierra, León: Universidad de León.

3 La única salvedad reseñable es el trabajo de Fernando García González. Vid. GARcía GonZÁLEZ, F. (1997). Mentalidade e cultura en Ferrol durante o século XVIII, Ferrol: Ateneo Ferrolán. A nivel gallego son de absoluta referencia los trabajos de Antón Costa y Ofelia Rey. REY Castelao, O. (2003). Libros y lectura en Galicia. Siglos XVI-XIX, Santiago de Compostela: Xunta de Galicia; Costa Rico, A. (2004). Historia da educación e da cultura en Galicia, Vigo: Edicións Xerais de Galicia.

4 Ruiz BerRio, J., (1998). "La educación del pueblo español en el proyecto de los ilustrados". Revista de Educación. pp. 163-192, p. 170.

${ }^{5}$ MuncK, T. (2001). Historia social de la Ilustración, Barcelona: Crítica, pp. 83 y ss. 
"domesticación" de unas clases populares excesivamente proclives al vicio y la inobediencia. El corresponsal ferrolano de Nifo para su "Correo general", cuando se quejaba de la carencia de centros formativos para la juventud en la Real villa, afirmaba tajantemente que de esta circunstancia provenían "innumerables desórdenes, porque donde no hai educación bien regulada están sobervias las costumbres, por no reconocer el yugo de la ley, ni el freno de la obediencia" ${ }^{\prime 6}$. La instrucción era pues considerada como el mejor y más seguro medio para alcanzar el estado virtuoso en los individuos, que era lo mismo que garantizar un pueblo dócil y fácil de gobernar. Ahora bien, los beneficios de la educación no podían ser disfrutados en igual proporción por todos. En una sociedad tan marcadamente escalonada, en buena lógica habría de existir también un escalonamiento en el acceso a los bienes culturales. Por tanto, solamente una minoría selecta podría alcanzar las exigentes propuestas del nuevo modelo. El propio Jovellanos, ardiente defensor de la educación universal, advertía del peligro que podía tener para el equilibrio de la sociedad la igualación en los saberes. Por ese motivo, defendía su limitación a niveles elementales en los hijos de las clases populares, es decir, a la enseñanza de lo simplemente necesario para su capacitación técnica en el mercado de trabajo. También el conde de Campomanes, se conformaba con que los hijos de los artesanos adquiriesen unos firmes conocimientos cristianos, así como de lectura, escritura, matemáticas y dibujo. El mucho más pragmático conde de Floridablanca, se identificaría plenamente con las opiniones del corresponsal ferrolano de Nifo, puesto que para él, la educación no era más que un instrumento de profilaxis social, un eficaz medio para acabar con la ociosidad de las clases menos favorecidas. La educación pues completaría y complementaría la política estatal de creación de hospicios, hospitales y casas de misericordia, la organización de cuerdas de vagos o las condenas a galeras y arsenales de los criminales y los ociosos?

Dada la estrecha relación entre educación, paz social y progreso económico, era lógico que durante el Siglo de las Luces, la cuestión de la reforma del sistema educativo estuviese muy presente, tanto en las obras de pensamiento, como en los planes de los diferentes gabinetes borbónicos. Nunca hasta ese siglo se había

${ }^{6}$ Nifo, F. M. (1770). Correo general de España, para beneficio común de los labradores, artesanos, comerciantes, e industriosos: para instrucción y gloria de los aficionados y profesores de ciencias y para entretenimiento de curiosos y políticos, Madrid, p. 261.

${ }^{7}$ Martínez Shaw, C. (1996). El Siglo de las Luces. Las bases intelectuales del reformismo, Madrid: Temas de Hoy, p. 96; Álvarez-Uria, F. (1998). "La Ilustración y su sombra. Dominación cultural y pedagogía social en la España del Siglo de las Luces". Revista de Educación pp. 347-372, p. 355 . 
generado una coyuntura tan favorable para el desarrollo de la reforma de un sistema que se consideraba obsoleto. A lo largo de la centuria proliferaron planes y proyectos educativos y se desarrolló una corriente de opinión favorable a los cambios a través de la prensa, los discursos o las sociedades económicas de amigos del país. El enfoque utilitarista que se daba a la educación permitió el desarrollo de la idea de la universalización de la enseñanza, obviamente, con las inevitables diferencias entre clases y $\operatorname{sexos}^{8}$. Al mismo tiempo, fue ganando peso la opinión de que la Iglesia no podía monopolizar la educación de los súbditos, al preocuparse más por crear buenos cristianos que individuos verdaderamente útiles al Estado? Por este motivo se pretendía sustituir los viejos criterios de caridad y beneficencia por la idea de la utilidad pública de la educación, mucho más acorde con el pensamiento ilustrado ${ }^{10}$. No obstante, estas pretensiones no deben interpretarse como un ataque directo a la religión, que se consideraba instrumento básico para la formación del individuo, sino más bien como un intento de frenar la excesiva influencia de la Iglesia en la enseñanza.

Mientras se iba fraguando ese clima favorable entre la opinión pública, o mejor sería decir, entre la minoría intelectual, la Corona durante el reinado de Carlos III, comenzó a desarrollar los primeros proyectos, cuya finalidad última, en lo que se refiere a la enseñanza primaria, era poner al menos un cierto orden en aquel caótico mundo. Uno de los principales objetivos planteados fue la reforma de la formación y condición de los maestros de primeras letras. La Real Cédula de 3 de octubre de 1763, cuyo ámbito de acción en un principio era la villa y corte, exigía del educador una dedicación exclusiva. Unos años después, en julio de 1771, la Corona fijaba por primera vez a nivel nacional los requisitos que debía reunir los maestros, además de liberar al oficio de buena parte de los impedimentos que podían perturbar su consideración social. La tendencia secularizadora de la educación se manifestó en la Real Provisión de 5 de octubre de 1767, en la que se afirmaba que los religiosos "jamás pueden competir con los maestros y preceptores seglares que por oficio e instituto se dedican a la enseñanza y procuran acreditarse al atraer discípulos y mantener con el producto de su trabajo a su familia”. Cuatro años más

${ }^{8}$ RuIZ BERRIO, J. (1998). “La educación del pueblo español...”, p. 171.

9 Delgado Criado, B. (1992). "Política educativa”. En Delgado Criado, B. (Coord.). Historia de la educación en España y América. La educación en la España Moderna (siglos XVI-XVIII), Madrid: Fundación Santa María, pp. 649-657, p. 649; MAYORDOMO, A. (1998). "Iglesia, religión y Estado en el Reformismo Pedagógico de la Ilustración Española”. Revista de Educación, pp. 445-466, p. 445.

10 Iglesias, Ma ${ }^{\mathrm{a}}$. C. (1990). "Educación y pensamiento ilustrado". En, Actas del Congreso Internacional sobre Carlos III y la Ilustración, Madrid: Ministerio de Cultura, Vol. III, pp. 1-30, p. 23. 
tarde, una disposición real declaraba sin valor para la obtención del bachiller en artes, los cursos realizados por los regulares en sus propios conventos ${ }^{11}$. Por otra parte, se intentó también desarrollar una política de uniformización del sistema educativo en todos los puntos de la Península, a través de la organización de los contenidos a impartir, la implantación de los catecismos o catones y la aprobación de determinadas normas sobre educación femenina y "población marginal escolar" ${ }^{\prime 2}$. Dentro de esta línea reformista, la disposición regia más conocida fue la Real Cédula de 12 de julio de 1781 sobre la obligatoriedad de la enseñanza, más declaración de intenciones que norma de verdadera efectividad. Mucho más explícita fue, sin duda, la ley 41 de las Cortes de Navarra de 1780-1781, en la que con meridiana claridad se declaraba obligatoria y gratuita la enseñanza escolar de niños y niñas comprendidos entre los cinco y los doce años de edad. Estas disposiciones, sobre todo la del territorio foral, seguían la estela de las vanguardistas normas aprobadas ya por otros estados europeos, como Prusia o Austria.

A pesar de todo lo expuesto, parece hoy asumido que aún siendo más que notable la proliferación de escritos y legislación sobre el tema, ni siquiera durante el reinado del más ilustrado de los monarcas, Carlos III, existió un verdadero plan de reforma educativa a nivel global. Solamente los estudios universitarios contaron con un verdadero proyecto, ideado por Mayans que, por otro lado, nunca llegó a implantarse. Además, lo cierto es que prácticamente todas las medidas tomadas se convirtieron en mero papel mojado, manteniéndose la educación primaria en un estado auténticamente lamentable, tanto en lo que respecta a los planes y métodos de docencia como a la propia financiación del sistema ${ }^{13}$. De lo que sí puede hablarse, no obstante, es de un cambio de mentalidad desde el reinado carolino en adelante, requisito indispensable para el posterior desarrollo reformista. Un cambio de mentalidad paulatino y no uniforme, puesto que como en otros tantos aspectos de la vida política, social, económica o cultural, el estallido de la Revolución Francesa y las dramáticas consecuencias de la Guerra de Independencia, supusieron un inevitable freno a ese proceso. No obstante, por primera vez el Estado actuó como único y legítimo monopolizador de la enseñanza frente no sólo a la Iglesia, sino también a los intereses gremiales y a los de la nobleza

11 VARela FernÁndez, J. (1998). "La educación Ilustrada o cómo fabricar sujetos dóciles y útiles”. Revista de educación, pp. 245-274, p. 264.

12 Ruiz Berrio, J. (1998). "La educación del pueblo español...", p.174; IGLESIAS, Ma.C. (1990). "Educación y pensamiento...", p. 24.

${ }^{13}$ Delgado CRiado, B. (1992.). Historia de la educación..., p. 649. 
conservadora. Los planes educativos del Estado liberal serán, pues, deudores de este importante primer paso acometido por los ilustrados.

\section{PROBLEMAS Y SOLUCIONES A LA DEMANDA DE EDUCACIÓN EN EL FERROL DIECIOCHESCO}

Dada la escasa incidencia de las medidas legislativas en materia de educación y a pesar del creciente interés del Estado, la Iglesia y los particulares continuaron monopolizando durante todo el Antiguo Régimen el sistema educativo primario, tanto en lo que respecta a iniciativas como a financiación. Junto a ellos, los municipios jugaron también un papel importante en muchas localidades de la Corona de Castilla, aunque parece que en la Galicia occidental su concurso fue bastante más limitado ${ }^{14}$. Sea como fuere, en el caso específico ferrolano, el municipio participaba activamente en la financiación de una escuela de primeras letras, al menos desde comienzos del siglo XVII ${ }^{15}$. Sin embargo, el acelerado crecimiento de la villa a partir de la década de los cincuenta del XVIII, hacía necesaria la creación de un centro de estudios primarios acorde con las nuevas dimensiones de la capital de departamento. Lamentablemente, las estrecheces por las que pasaban las arcas municipales, unidas al criterio de sus alcaldes mayores de dar prioridad a otras necesidades básicas de la población, frenaron cualquier intento de mejora del sistema educativo público ferrolano hasta bien entrada la década de los ochenta. El único ensayo serio hasta aquellas fechas se produjo en 1773, cuando el consistorio intentó adquirir un terreno a propósito "para hacer en él oficina para la escuela de primeras letras", sin resultado efectivo alguno ${ }^{16}$. Como en tantos otros aspectos de la vida ferrolana, fue la impecable acción de gobierno del alcalde mayor D. Eugenio Manuel Álvarez Caballero la que sacó a la villa de la precariedad que sufría en materia educativa. En 6 de septiembre de 1785, el magistrado obtenía del Consejo de Castilla la creación de las cátedras de gramática y primeras letras "en beneficio de las mucha juventud de la villa". La escuela de primeras letras estaría a partir de entonces bajo la dirección de un maestro de leer, escribir y contar, con una asignación anual de 3.000 reales, a cobrar de los fondos del arbitrio del vino. Para auxiliarle en esta tarea, contaba con el concurso de un ayudante, que recibía anualmente 1.500 reales. Además de dicho sueldo, tanto el maestro como su ayudante contaban al año con veinte días de asueto, a cumplir, en

14 SANZ GonZÁlEZ, M. (1992). “Alfabetización y escolarización en Galicia a fines del Antiguo Régimen”. Obradoiro de Historia Moderna, pp. 229-249, p. 232.

15 Montero Aróstegui, J. (1972). Historia y descripción de El Ferrol, Pontedeume (1 ${ }^{\text {a }}$ Ed. Madrid 1859), p. 290.

${ }^{16}$ A.M.F. (Archivo Municipal de Ferrol), Libro de consistorio $n^{\circ} 10$ (1771-1775), fol. 167. 
el caso del primero, durante los meses de verano y del segundo, "desde Pascua de Resurrección hasta de Espíritu Santo"17.

Con las dotaciones estipuladas, maestro y ayudante estaban obligados a enseñar gratuitamente a todos los educandos pobres. Los pudientes debían de pagar una mensualidad, de todos modos, poco onerosa: hasta comienzos de 1788 se cobraba por la enseñanza de escritura y cuentas tres reales y por la de lectura un real y dieciocho maravedíes. A partir de entonces, al entrar en funcionamiento el nuevo edificio destinado a escuela y, por consiguiente, dejarse de pagar el alquiler mensual de cincuenta reales del viejo local, los precios se rebajaron a dos y un real respectivamente ${ }^{18}$. El dinero ingresado por las cuotas de los alumnos, según la instrucción de 19 de octubre de 1788, debía repartirse "entre los dos con la misma igualdad" 19 .

Comparando la asignación anual que recibía tanto el maestro ferrolano como su ayudante con la situación salarial de los docentes de primeras letras en la Galicia de la época, parece constatarse un mayor desahogo económico en el caso de los departamentales. Frente a los 3.000 y 1.500 reales que recibían anualmente uno y otro, la media calculada por Margarita Sanz González para la zona sudoccidental gallega no llegaba siquiera a superar los 113. Algo mayor era en el caso del vecino principado de Asturias, a juzgar por las estimaciones del profesor Barreiro Mallón 345,5 reales anuales ${ }^{20}$-, pero, aún así, las distancias con respecto a Ferrol continuaban siendo muy considerables. La incidencia del mundo rural, en donde la enseñanza de las primeras letras estaba francamente muy mal pagada, explican en parte esos raquíticos salarios medios. De todos modos, los mayores ingresos tanto en el actual provincia de Pontevedra como en Asturias, se hallaban también muy por debajo de los ferrolanos: en el caso pontevedrés, solamente eran localizados para mediados del siglo XVIII dos maestros con ingresos superiores a los 400 reales, mientras que en Asturias el docente más acomodado apenas alcanzaba los 800 al año. Sin embargo, efectuando esta comparación exclusivamente con otros centros urbanos, las diferencias desaparecen, mostrándose el salario de los maestros ferrolanos incluso un tanto por debajo de la media. Así sucede si

${ }^{17}$ Ese año, el ayudante Manuel de Villarnobo solicitará del ayuntamiento el cambio de esos días libres al mes de junio. A.M.F., Libro de consistorio $n^{\circ} 16$ (1788), fol. 181.

18 A.M.F., Libro de consistorio $n^{\circ} 16$ (1788), fol. 148.

19 A.M.F., Instrucción pública, Caja 774.

20 SANZ GONZÁLEZ, M. (1992). “Alfabetización y escolarización...”, pp. 236-237; BARREIRO MALlón, B. (1998). "Alfabetización y lectura en Asturias durante la Edad Moderna”. Espacio, Tiempo y Forma. Historia Moderna, pp. 115-134, p. 120. 
efectuamos el pertinente contraste con los resultados obtenidos en las ciudades de Toro y Zamora para el último tercio de siglo. En ellas, los maestros de escritura los equivalentes al titular ferrolano- cobraban 3.300 reales y los de lectura - los equivalentes al ayudante- 2.750 y 1.650 respectivamente. En la Palencia de 1800, alcanzaba el primero los $4.400 \mathrm{y}$ el segundo $\operatorname{los} 2.750^{21}$ y en la propia Villa y Corte, los maestros de las ocho Escuelas Reales creadas en 1791, cobraban la misma cantidad que el palentino de escritura ${ }^{22}$. En suma, si bien los salarios de los maestros titulares ferrolanos eran considerablemente superiores al entorno rural más inmediato, no pueden ser considerados ni mucho menos como excesivos, teniendo en cuenta además la fuerte inflación que padecían los vecinos de la real villa, sin duda muy superior a los precios de los productos de primera necesidad en ciudades bastante menos dinámicas como, por ejemplo, las zamoranas. Afortunadamente, ambos contaban con el auxilio de las asignaciones de los alumnos pudientes que incrementaban en algo el fijo económico garantizado por los fondos de arbitrios y que fueron el fruto de la discordia entre el maestro y sus distintos ayudantes a lo largo del último tercio del siglo XVIII.

Si bien en un primer momento se dejaba al criterio del maestro titular de la villa la exigencia o no a los alumnos del pago mensual, pronto el municipio intentó perfilar más nítidamente el concepto de estudiante pobre. Éste debía extenderse no sólo a los hijos de personas sin recursos económicos, sino también a los de aquellos jornaleros que trabajando en las instalaciones reales, sus sueldos no llegaban a los treinta cuartos, pues "en un país tan caro apenas a muchos alcanzan para el pan, alimento de primera nezesidad". El 19 de febrero de 1797, D. Ambrosio de Moya, ayudante de la escuela pública, quiso ir un poco más allá, proponiendo al concejo la gratuidad absoluta de la enseñanza de la lectura en el centro, renunciando a la gratificación que obtenía por cada alumno. Justificaba esta medida por el importante crecimiento de la población ferrolana desde las instrucciones de Álvarez Caballero, así como por la existencia de "mucha más gente pobre y jornalera, de suerte que la maior parte abandona a sus hijos a una crianza idiota y sin cultura por no tener escuela gratuita donde llevarlos, estando llenas las calles de estos niños desgraciados". La propuesta del ayudante fue rápidamente tomada

${ }^{21}$ Lorenzo PinaR, F.J. (1997). La educación en Zamora y Toro durante la Edad Moderna. Primeras letras y estudios de Gramática, Zamora: Semuret, pp. 29 y 45; GUITIÉRREZ BARBA, A. (1990). "Una aproximación a la enseñanza pública palentina de las primeras letras en el tránsito del siglo XVIII al XIX” En, Actas del II Congreso de Historia de Palencia, Palencia: Diputación de Palencia, Vol. V, pp. 717-729, 722.

${ }^{22}$ Aguilar PiÑAl, A. (1987). "La política docente”. En, Menéndez Pidal, R. Historia de España, Madrid: Espasa, T. XXXI, pp. 439-484, p. 447. 
como propia por el síndico procurador de la villa que en marzo de aquel mismo año, presentaba un informe tremendamente crítico con la gestión del maestro titular, D. Jacobo Violán y Romero, cuyo proceder, era calificado sin ambages como "criminal". Se acusaba al docente de olvidarse de la educación de los niños pobres, convirtiendo a la escuela pública en "un destino formado solo para lucro y enriquecimiento del actual maestro, no para alivio de sus pobres vecinos". Como dato elocuente del interesado proceder de Violán, el procurador presentaba una relación de los alumnos, donde se apreciaba que frente a los veinte que no pagaban, había ciento treinta que sí lo hacían. Para evitar la tentación del enriquecimiento personal, abogaba pues por la entera gratuidad de la enseña en la escuela de la villa, medida que, a su entender, beneficiaría a unos cuatrocientos cincuenta hijos de jornaleros "infelices y desnudos, a quienes vemos abandonados por las plazas y calles sin más destino que una perniciosa ociosidad" ${ }^{23}$. Los hijos de los pudientes no tenían por qué experimentar ningún perjuicio, puesto que podían estudiar en alguna de las siete escuelas particulares que existían en la plaza por aquellos años. Además, recomendaba un mayor control por parte del municipio tanto en la admisión de niños, con la introducción de unas papeletas que debían contar con la firma de un delegado municipal, como en la inspección de los progresos operados. Por último, estimaba conveniente la desaparición del cargo de ayudante, sustituyéndose por una segunda plaza de maestro, dedicada a la enseñanza de la lectura y la doctrina cristiana y enteramente independiente del control del maestro principal, que se encargaría por su parte de la enseñanza a los alumnos más mayores de la escritura y las matemáticas. El cambio de categoría del maestro no habría de repercutir en su sueldo, que seguiría estipulado en los 1.500 reales fijados por Álvarez Caballero, aunque, en contrapartida, tendría la posibilidad de ascender al primer cargo en caso de producirse la vacante.

El plan de reforma del síndico no se puso en práctica, pero refleja perfectamente uno de los principales problemas a los que tuvo que enfrentarse el consistorio durante décadas: las tensiones entre el maestro titular y sus distintos ayudantes. Sin duda, la peculiar personalidad de D. Jacobo Violán, tuvo mucho que ver en estos enfrentamientos, dada su rapacidad a la hora de querer acaparar buena parte de los ingresos económicos que aportaban los alumnos. Aún así, también el a veces excesivo afán de independencia del ayudante con respecto a su superior, tanto en materia educativa como de disciplina en la escuela, tuvo mucho que ver en el incremento de la conflictividad. Aunque D. Jacobo tuvo problemas con varios de sus subalternos, sin duda, las tensiones más agudas las vivió en las últimas décadas

${ }^{23}$ A.M.F., Instrucción pública, Caja 774. 
del siglo XVIII con Manuel de Villarnobo. Parece que en el fondo del asunto se encontraban los intereses económicos de ambos, en tanto en cuanto no se podían de acuerdo en las cantidades pecuniarias que habían de recibir cada uno. A ello se unía la resistencia del ayudante a obedecer los dictámenes del maestro, dado que en su opinión aquel se comportaba de manera tiránica.

\section{MAESTROS PARTICULARES Y MAESTROS ILEGALES}

Junto con la escuela sostenida por el municipio, la villa de Ferrol contaba con una serie de establecimientos particulares, mantenidos exclusivamente por las aportaciones económicas de los padres de los alumnos. El número de estos centros varió considerablemente a lo largo del siglo XVIII. En 1790, año en el que el ayuntamiento, por orden del Consejo de Castilla, elaboró una estadística de escuelas en la villa, existían abiertas junto a la pública, otras cinco: una regentada por D. Antonio de Puente y Andrade en el número 52 de la calle Real, la de Francisco Gamero en el barrio de Esteiro, la de Francisco Fernández en la Plaza Nueva, otra en el número siete de la calle de San Roque bajo la dirección de Tomás de la Carrera y la de Manuel Pérez en San Amaro. Las carencias económicas del vecindario ferrolano y la competencia desleal de individuos que ejercitaban la docencia sin el pertinente título y autorización del gobierno local, hacía que algunos de estos maestros, se sostuviesen con no pocas dificultades. La Real Provisión de 11 de julio de 1771, había reglamentado a nivel nacional los requisitos exigidos a los aspirantes al ejercicio del magisterio. En primer lugar, debían de presentar una certificación eclesiástica de haber sido examinados y aprobados de doctrina cristiana. También era requisito imprescindible adjuntar el preceptivo informe de la justicia en donde se acreditase su domicilio, buena vida, costumbres y limpieza de sangre. Una vez comprobadas estas informaciones, el aspirante se sometía a un examen sobre su "pericia del arte de leer, escribir y contar" ante dos comisarios del ayuntamiento y dos examinadores o veedores. Si lograba superar todas estas exigencias, se remitía toda la documentación a la Hermandad de San Casiano o, a partir de 1780, al Colegio Académico de Primeras Letras, a quienes correspondía aprobarla y elevarla al Consejo de Castilla para que expidiese el título correspondiente.

En el Ferrol del último tercio del siglo XVIII el municipio de la villa parece que cumplía obedientemente con estas disposiciones legales, tal y como nos manifiestan varios testimonios reflejados en la documentación estudiada. La única salvedad que debemos poner de manifiesto, está relacionada con el número de diputados municipales comisionados para la supervisión del examen, ocupándose de esos menesteres en Ferrol solamente uno, frente a los dos señalados en las disposiciones regias; los agobios del gobierno municipal para atender los múltiples 
problemas de un centro urbano que crecía a pasos agigantados, explica esta eliminación del segundo diputado que, por otro lado, no tuvo mayores repercusiones. Las muestras de la actuación del concejo departamental en los exámenes de aspirantes a maestros de primeras letras, son lo suficientemente importantes como para poder hacernos una idea bastante aproximada del funcionamiento de unos mecanismos de control de la calidad de la enseñanza escrupulosamente acordes con la legalidad vigente en la época. Por ejemplo, en abril de 1788, tras recibir la petición de un individuo para abrir escuela de primeras letras, acordaba que el maestro titular de la villa junto a su ayudante, con la presencia de un diputado nombrado por el municipio, examinase al aspirante "tanto en leer como en escribir y contar hasta las cinco reglas" ${ }^{24}$. El 24 de julio de ese mismo año, el consistorio recibía una nueva solicitud, firmada por un tal D. Francisco García. En esta ocasión, la ausencia de parte los requisitos documentales exigidos, impidió al aspirante obtener la gracia del examen al que quería enfrentarse, al menos hasta que completase correctamente toda la información requerida ${ }^{25}$.

Los ejemplos hallados no se circunscriben exclusivamente a la década de los ochenta: en junio de 1794, D. Sebastián de Hevia, solicitaba del municipio la autorización para la apertura de una escuela de primeras letras en el barrio de Esteiro. El regimiento, cumpliendo de nuevo con las normas legales establecidas, encomendó a dos maestros de la villa junto al regidor de policía para que valorasen su pericia en la escritura, lectura y matemáticas a través de un examen ${ }^{26}$. Este panorama pervivirá en Ferrol hasta la creación a comienzos del siglo XIX de las Juntas de exámenes, que poco a poco irá apropiándose de la capacidad de la Academia para examinar a los maestros españoles ${ }^{27}$. Pero aún existiendo una normativa legal para regular la concesión de escuelas de primeras letras, fueron abundantes las quejas de los maestros de la villa ante la intromisión en su trabajo de individuos sin licencia, que limitaban considerablemente el ya de por si estrecho mercado de la enseñanza primaria. El 18 de enero de 1786, el maestro titular de la villa, D. Jacobo Violán, en un informe elaborado por orden del alcalde mayor Álvarez Caballero, se quejaba ante el magistrado del absoluto descaro con el que

${ }^{24}$ A.M.F.,Libro de consistorio $n^{\circ} 16$ (1788), fol. 20.

25 A.M.F., Libro de consistorio $n^{\circ} 16$ (1788), fols. 55 y 55 vto.

${ }^{26}$ A.M.F.,Libro de consistorio $n^{\circ} 20$ (1794-1796), fol. 61.

${ }^{27}$ VARELA, J. (1998). "La educación Ilustrada o cómo fabricar...”, p. 264; RUIZ BERRIO, J. (1998). “La educación del pueblo español...”, pp. 174-178. 
los aforados castrenses ejercían la docencia, con notorio desprecio tanto de las disposiciones concejiles como de la propia legislación real $^{28}$.

En esa ocasión y como medio para escabullirse de las posibles interferencias del alcalde mayor, habían optado por situar la escuela fuera del ámbito jurisdiccional de la plaza. Sin embargo, en no pocos casos, los aforados castrenses abrían sus escuelas dentro de aquel territorio, sin que el alcalde mayor y sus colaboradores pudieran hacer nada para evitarlo, tanto por la inmunidad de los infractores, como por la propia incapacidad del municipio, dada la carestía de fondos y personal que padecía. Solamente un año después del mencionado informe del maestro titular, tanto él como su ayudante, en una visita a las escuelas de la villa, volvían a incidir en la gravedad del problema, al constatar el escaso número de alumnos que recibían clases en las escuelas permitidas "demanado de que en los arsenales ay sugetos que se dedican a la enseñanza en contravención de la Real Zédula de 11 de julio de 71 , y de que varios soldados de artillería y marina dan lecciones por las casas" ${ }^{\prime 2}$. Una situación muy similar se estaba produciendo en la vecina villa de A Graña, localidad también con una nutrida población de fuero castrense y que se hallaba bajo el gobierno político del alcalde mayor ferrolano: en septiembre 1793, D. Manuel Crespo Valles, su maestro de primeras letras, denunciaba a un tal Miguel Sanguiño "individuo de la brigada retirado" por ejercer la profesión sin licencia. A pesar del compromiso del ayuntamiento por atajar el problema, un año más tarde el maestro de A Graña volvía a quejarse de la impunidad con la que el militar retirado ejercía dicho oficio. En octubre de ese mismo año, los maestros de Ferrol volvían a dirigirse al alcalde mayor por problemas similares en la real villa. Poco después, en abril de 1794, la denuncia se personificaba en dos individuos de la Armada Real, los miembros de las brigadas José López y Francisco Maza ${ }^{30}$. Ante estos atropellos, la actitud de las autoridades de Marina fue siempre muy permisiva con sus aforados, a pesar de que en algunos momentos puntuales, los intendentes buscasen maquillar esa actitud con ciertos gestos de acercamiento, más como vehículo para evitar los siempre engorrosos conflictos de competencias que buscando sinceramente acabar definitivamente con ellos.

Aunque buena parte del problema de la enseñanza ilegal estaba relacionado con la actividad de los aforados, no siempre eran éstos los responsables del nacimiento de nuevas escuelas al margen de la ley. El 17 de noviembre de 1796, los maestros

\footnotetext{
${ }^{28}$ A.M.F., Instrucción pública, Caj. 774.

${ }^{29}$ A.M.F., Libro de consistorio $n^{\circ} 16$ (1788), fol. 169.

${ }^{30}$ A.M.F., Libro de consistorio $n^{o} 19$ (1791-1793), fol. 250; Libro de consistorio $n^{\circ} 20$ (17941796), fols. 52 y 54; Libro de consistorio $n^{\circ} 20$ (1794-1796), fols. 52 y 54.
} 
de la villa, denunciaban ante el corregidor a Salvador Iglesias "mozo soltero de corta edad y ninguna ciencia", por haber "establecido arbitrariamente por solo su antojo y sin obtener el correspondiente diploma escuela de primeras letras, mezclando indebidamente ambos sexos y no sólo esto, sino que como no tiene la instrucción y talento preciso, está dando una educación y enseñanza monstruosa, de que se infieren infinitos perjuicios al Estado y buenas costumbres", aparte de perjudicar, claro está, los intereses de los maestros. El consistorio, en esta ocasión, acordaba obligarle a cerrar su escuela, amenazándole con dos meses de prisión en caso de volver a las andadas. Mucho más importante que la acción de algún personaje como Iglesias, es la importante actividad docente de las mujeres. En teoría, y aplicando escrupulosamente la ley, las maestras sólo podían ejercer la docencia con las integrantes de su sexo pero, aprovechándose del caótico estado de la política educativa de la villa, muchas de ellas acogían a niños en sus establecimientos, casi siempre ilegales, con el consiguiente escándalo de los maestros, manifestado en un sin fin de memoriales dirigidos al consistorio que no sirvieron más que como justificado recurso al pataleo.

El problema de las escuelas ilegales siguió estando muy presente en la primer tercio del siglo XIX. En septiembre de 1810, el procurador general de la villa, D. Agustín Cortiñas, informaba al consistorio del flagrante incumplimiento de las normativas municipales por parte de un número importante de maestros y maestras. Éstos, a pesar de haberse superado ya el plazo de un mes que se había establecido como ultimátum, no sólo no habían acudido a efectuar el examen estipulado, sino que continuaban ejerciendo su oficio con total impunidad. En diciembre, los maestros legales volvían a elevar un memorial a las autoridades municipales quejándose de las escuelas regentadas por aforados castrenses y mujeres. Cinco años más tarde, D. Jacobo Violán presentaba un memorial al gobernador de la plaza en el que volvía a incidir en los problemas que ya había señalado casi treinta años antes en el informe ordenado elaborar por Álvarez Caballero; sin embargo, en esta ocasión, al ya tradicional ejercicio de los militares se unía la actividad de "hombres acabando de salir del presidio de estes arsenales por falsarios". El maestro se refería a Felipe Alvacastro, presidiario de los arsenales que incluso antes de finalizar su condena, había abierto ya en San Amaro una pequeña escuela en donde enseñaba a niños de ambos sexos. Junto a ese escandaloso caso, el ya veterano maestro titular había localizado otras siete escuelas ilegales, casi todas vinculadas con militares en ejercicio. De entre ellos destacaban sobremanera los integrantes de las Brigadas de Marina. A este cuerpo pertenecían tanto el sargento Manuel García, que tenía abierta escuela en las inmediaciones de la parroquia de San Fernando, como otros tres colegas, que contaban con las suyas 
respectivamente en la calle de San Benito y las plazas de Armas y Angustias ${ }^{31}$. El problema persistía en 1826, cuando el consistorio contabilizó de nuevo ocho maestros intrusos, entre los que figuraba el propio Felipe Alvacastro.

La competencia desleal de los ilegales, unida a una muy poco disimulada actitud gremial, propició la continuada exigencia de los maestros para que el regimiento limitase el número de escuelas en la villa. El alcalde mayor Álvarez Caballero accedió, en enero de 1786, a tal pretensión, dividiendo la población escolar entre un total de cuatro escuelas: una en Esteiro bajo la dirección de D. Antonio de Puente y Andrade, otra en Ferrol Viejo, encomendada a Francisco Fernández, la de Canido, con Tomás de la Carrera al frente, encargándose la escuela de la villa con D. Jacobo Violán y su ayudante, de la instrucción del resto del pueblo. El magistrado estipulaba además la prohibición de la apertura de ningún otro centro de este tipo en toda la jurisdicción real, exceptuando el hecho de que alguno de ellos dejase la plaza de maestro vacante. Curiosamente, el alcalde mayor permitió que continuase su actividad la escuela abierta en la puerta de Caranza, y que tantas quejas había generado, sobre la condición de que siempre estuviera "sujeta a las reglas que para el adelantamiento de la jubentud están prescritas". En correspondencia con este privilegio legal que les otorgaba de facto el monopolio de la educación de primeras letras en la villa, los maestros habían de admitir en sus escuelas a algunos niños pobres, amén de cumplir unas instrucciones generales que elaboraría el maestro titular de la villa, con el necesario visto bueno de Álvarez Caballero ${ }^{32}$. Las instrucciones de 1786 , pese a su buena intención y al indudable efecto positivo que habrían tenido en la caótica organización escolar ferrolana, no llegaron a cumplirse. Desde luego, el eterno problema de las escuelas ilegales, no desapareció con estas disposiciones. Existió además cierta resistencia por parte de algunos maestros a trasladar su centro de estudios de lugar. Ese fue el caso de D. Antonio de Puente y Andrade, docente que tenía abierta una escuela en plena calle Real y que no quería cambiar su selecta clientela por los hijos de los operarios de la maestranza residentes en Esteiro, a donde le había tocado en suerte trasladarse. Esas resistencias, unidas a la escasa capacidad del municipio para hacer cumplir las normas precipitaron un fracaso que redundó en un cierto cambio de actitud de las autoridades políticas a la hora de conceder nuevas licencias de apertura de escuelas, pese a la alborotada respuesta de los maestros de la villa.

${ }^{31}$ A.M.F., Instrucción pública, Caj. 774.

32 A.M.F., Libro de consistorio $n^{o} 16$ (1788), fol. 169; Instrucción pública, Caj. 774. 


\section{MATERIAS Y MÉTODOS DE ENSEÑANZA}

Para introducirnos en los métodos pedagógicos de las escuelas de primeras letras ferrolanas durante el XVIII, contamos con la inestimable ayuda del informe elaborado por el ayuntamiento en 1790, por orden del Consejo de Castilla, cuya misión era calibrar la calidad de la enseñanza en todo el país. En consecuencia, a lo largo de aquel año, todos y cada uno de los maestros de la villa entregaron al municipio su preceptiva memoria, en donde señalaban, con mayor o menor profusión dependiendo del caso, su modo de trabajar con los pupilos, amén de las materias de enseñanza que se ofrecía en sus escuelas. Curiosamente, el documento más parco es el del maestro titular de la villa, sin duda debido a que su actividad docente se hallaba mucho más controlada por el municipio, tanto a través de la supervisión de sus diputados como por la estricta reglamentación elaborada en 1788 por Álvarez Caballero. La documentación emanada de dicha averiguación se halla un tanto dispersa en el propio libro de consistorios de la villa, incorporándose a él a medida que llegaban los informes. Se trata de una fuente de gran riqueza en información, a pesar de la inevitable subjetividad que desprende. Teniendo en cuenta que fueron los propios maestros sus autores, es notoria la tendencia a una cierta idealización del método pedagógico empleado. Aún así, la práctica coincidencia de todos ellos en las líneas generales de la enseñanza a desarrollar con los alumnos, parecen remitirnos a unas altas dosis de verosimilitud.

La semejanza de los métodos empleados en las escuelas de la villa podría responder tanto a las propias características del sistema educativo español de la época como a las peculiaridades del caso ferrolano; nosotros, sin descartar completamente los efectos del segundo, pensamos que es el primer aspecto el que con mayor fuerza incidió en esta relativa homogeneidad. Es evidente que en la época, la educación primaria en toda España se hallaba en una lamentable situación, anclada en unos métodos verdaderamente caducos y con unos muy discretos objetivos que prácticamente en nada habían variado a lo largo de los siglos. Por ese motivo, no resulta extraño que en los análisis realizados para otros ámbitos territoriales, nos encontremos con un panorama tan similar al que aquí presentamos. Esto no quiere decir que no puedan existir en el caso ferrolano ciertas peculiaridades vinculadas a un tímido intento del municipio de poner cierto orden en la endeble estructura de la educación primaria de la villa. Sin duda, las famosas instrucciones de Álvarez Caballero tenían ese objetivo, al colocar a todos los centros educativos de la plaza, bajo la directa supervisión del maestro titular. Pero, aunque las fuentes son excesivamente parcas al respecto, parece que estas disposiciones tuvieron escaso cumplimiento, ante la tenaz resistencia de los maestros a la intromisión del concejo en sus labores. 
La primera conclusión importante que se desprende de la lectura de los diferentes informes elaborados por los maestros ferrolanos es la importancia de primer orden que jugaba en los estudios primarios la formación religiosa. Si durante el Medievo la enseñanza de la doctrina cristiana había permanecido al margen del sistema educativo, sin existir apenas diferencia entre la catequesis a adultos y a niños, a partir de la Época Moderna se integrará en las escuelas ${ }^{33}$. La Reforma católica emanada del Concilio de Trento, tomó conciencia del poder de la educación. Si bien es cierto que los mandatos de sínodos y concilios provinciales exhortando a los párrocos a la enseñanza de la doctrina cristiana y al control de la ortodoxia de los maestros ya eran habituales a finales del siglo XV, se intensificaron significativamente a partir de la segunda mitad del XVI, cobrando un nuevo impulso en el XVIII de la mano de los obispos reformistas ${ }^{34}$. La Corona y la Iglesia recalcaban la importancia de la escuela como vehículo de aprendizaje de la doctrina cristiana $\mathrm{y}$, de hecho, ésta se convirtió durante los siglos de la Edad Moderna en uno de los instrumentos más eficaces de moralización, inculcando a los niños los principales fundamentos de la fe católica ${ }^{35}$. Incluso escritores críticos con el sistema, como es el caso de Pablo de Olavide, defendían ardientemente la existencia de una educación infantil "ilustrada pero cristiana"36. En su informe al Consejo de Castilla en 1790, D. Jacobo Violán y Romero, maestro de la escuela principal de la villa, aseguraba que "siempre ha tenido este punto por el más principal en el cumplimiento de su magisterio, con el conocimiento de que importa poco el que a los niños se les instruya con la mayor perfección en las primeras nociones de las letras, si no se les enseña a ser buenos christianos"37.

Tanto en la escuela pública como en las privadas se iniciaban las tareas matutinas con una oración. En la información aportada por D. Jacobo Violán se indicaba que todas las mañanas, antes de comenzar con el estudio, los alumnos daban gracias a Dios "por tener otro día más de vida". Junto a la oración al comienzo de las clases, también se efectuaban las pertinentes plegarias a la salida. Era también común, generalmente a la tarde, el rezo del rosario, devoción-

${ }^{33}$ LASPAlas PÉRez, F.J. (1993). La reinvención de la escuela. Cinco estudios sobre la enseñanza elemental en la Edad Moderna, Pamplona: Eunsa, p. 53.

34 ViÑaO Frago, A. (1992). “Alfabetización, lectura y escritura en el Antiguo Régimen”. En, Escolano, A. (dir.), Leer y escribir en España. Doscientos años de alfabetización, Madrid: Pirámide, pp. $45-68$, p. 50.

35 ARIÈs, Ph. (1987). El niño y la vida familia en el Antiguo Régimen, Madrid: Taurus, p. 122.

36 VIÑAO FRAGO, A. (1992). "La influencia de Campomanes, Olavide y Cabarrús en la educación”. En, Delgado Criado, B. (Coord.), Historia de la educación... pp. 657-668, p. 663.

${ }^{37}$ A.M.F., Libro de consistorio $n^{\circ} 18$ (1790), fol. 169 vto. 
estandarte de la reforma católica tridentina. En las escuelas de dotación de la villa se rezaba junto con las letanías de la Virgen "pidiendo a Dios por la salud de sus majestades y por su feliz govierno". En la regentada por Francisco Fernández se hacía también todos los días, al igual que en la de D. Antonio de Puente y Andrade, situada en la calle real, que se rezaba ante una imagen de la Inmaculada, declarada patrona de la escuela y a la que se le dedicaba anualmente una novena. En la de Esteiro, no se rezaba el rosario en invierno, siendo sustituido por "algunas devociones oportunas" pero, en cambio, su maestro procuraba que los alumnos participaran en el rezo del Via Crucis que él mismo dirigía todos los domingos en la capilla castrense $\mathrm{e}^{38}$. Por su parte, el alcalde mayor Álvarez Caballero había estipulado en sus instrucciones de 1766 que los maestros de la villa además "de hacer que los muchachos capaces confiesen los domingos de minerba y comulguen por la mañana y de tarde", debían juntarse con todos sus alumnos, por estricto orden de antigüedad y presididos por el maestro titular de la villa, para "cantar el Rosario y doctrinas por el pueblo", con cuya alabanza "a el Rey de los reyes, (se) haga conocer el amor de sus hijos y estimule este piadoso exercicio de jóbenes sencillos a la edificación de adultos relaxados"39. Cuatro años más tarde, en mayo de 1790, el ayuntamiento siguiendo esta línea de potenciación de este ejercicio obligaba a todos los maestros de la villa a que saliesen con sus alumnos por las calles rezándolo ${ }^{40}$.

Todas las escuelas procuraban también fomentar la asistencia de los alumnos a la eucaristía. En la escuela principal, D. Jacobo Violán señalaba que a veces llevaba a los niños a escuchar la misa en la iglesia parroquial de San Julián, aunque esta actividad se realizaba "de raro en raro", dada la distancia existente entre el centro de estudios y el templo, lo que interrumpía en exceso el correcto desarrollo de las clases. En la de Esteiro, todos los días algunos niños voluntariamente acompañaban a su maestro a la misa de la parroquia de San Fernando. Al concluir el acto religioso, Francisco Gomero felicitaba a los que lo hacían con devoción y castigaba "por niño que sea" a aquellos que no estuvieran en la iglesia con la compostura debida ${ }^{41}$. Otra manifestación evidente del peso de la religión en la formación de la infancia se halla en la elección de patrones a los que se encomendaba la escuela. En el caso ferrolano, la advocación más común era la Inmaculada Concepción, cuya imagen presidía el salón de los ejercicios de la

\footnotetext{
${ }^{38}$ A.M.F., Libro de consistorio $n^{\circ} 18$ (1790), fols. 167 vto. y 169 vto.

${ }^{39}$ A.M.F., Instrucción pública, Caj. 774.

${ }^{40}$ A.M.F., Libro de consistorio $n^{\circ} 18$ (1790), fol. 41.

${ }^{41}$ A.M.F., Libro de consistorio $n^{\circ} 18$ (1790), fol. 167 vto. y 169 vto.
} 
nueva escuela pública. En su honor, en virtud de acuerdo del ayuntamiento de 21 de noviembre de 1805 , se celebraba una solemne función religiosa anual en la parroquia de San Julián, con la asistencia de todos los alumnos ${ }^{42}$. La Inmaculada también era patrona, al menos, de las escuelas de la calle real y Esteiro, mientras que la regentada por Tomás de la Carrera en el número siete de la calle de San Roque, estaba encomendada a Nuestra Señora del Socorro.

El sábado se dedicaba al estudio del catecismo y de la cartilla de buena crianza, a lo que se añadía, en el caso de la escuela de dotación de la villa, la enseñanza de la ayuda en la misa. El catecismo empleado para el estudio de la doctrina era, en todas las escuelas ferrolanas, el de Astete, variando simplemente la edición empleada. Éste, desde su primera edición de 1576, fue sin género de dudas, junto al de Ripalda, el texto más utilizado para estos menesteres en los territorios peninsulares de la Corona, manteniendo su vigencia mucho más allá de los límites de la Edad Moderna. Junto a él, en algunas escuelas privadas, como la de la calle real o la de Ferrol Viejo, se utilizaba el catecismo del padre Cayetano de San Juan Bautista, sacerdote de las escuelas pías. En la de Francisco Fernández, dado que, en su opinión, "el catecismo que regularmente es el que se estudia está tan diminuto en algunas cosas esenciales", el propio maestro había elaborado "un quaderno en que con brevedad está qué cosa es oración, qué es misa, quántas cosas son necesarias para confesar bien, qué es comunión y sus diferencias, qué circunstancias se requieren para comulgar dignamente y sus efectos" ${ }^{\prime 3}$. Fuera cual fuere el catecismo elegido, las diferencias en cuanto a estructura y, sobre todo, contenido eran muy sutiles. Todos ellos ofrecían un cuestionario o interrogatorio más o menos extenso con las principales bases dogmáticas del catolicismo, así como con las obligaciones de todo cristiano, fundamentadas en los mandamientos de la ley de Dios y de la Iglesia. Asimismo, los catecismos contenían las principales oraciones, comenzando por el padrenuestro y siguiendo con el avemaría, la salve o el credo. El método para aprender los rudimentos de la religión era la repetición y memorización de las oraciones, preguntas y respuestas en ellos contenidos, lo que llevó ya a ciertas críticas en el siglo XVIII, por ser más que de utilidad para los niños "instrucción propia para los papagayos" $"$. Junto a la repetición de las cuestiones planteadas en los catecismos, algunos maestros, empleaban como útil apoyo para fomentar la piedad y el temor al tremendo día del

\footnotetext{
42 Montero Aróstegui, J. (1972). Historia y descripción ..., p. 290.

${ }^{43}$ A.M.F., Libro de consistorio $n^{\circ} 17$ (1790), fol. 232 vto.

${ }^{44}$ LORENZO PINAR, F.J. (1997). La educación en Zamora y Toro..., p. 65.
} 
juicio, grabados y láminas. Así lo hacía, por ejemplo, Tomás de la Carrera, en su escuela de la calle de San Roque ${ }^{45}$.

El interés de la Corona por la evangelización de los niños respondía a su secular posición como paladina de la fe católica frente a herejes e infieles. Esa íntima identificación entre Trono y Altar servía asimismo para que la religión actuara como principal fundamento ideológico del sistema político establecido. Por eso, cuando el maestro Violán explicaba a sus alumnos las verdades de la fe, también les inculcaba "la obediencia que se debe tener a los soveranos (que representan a Dios)" ${ }^{\text {"46 }}$. A partir de la entrada en vigor en las escuelas de lo dictaminado por la Real Provisión de 11 de julio de 1771, junto al catecismo señalado por el ordinario de la diócesis, se debía enseñar en ellas a través del "compendio histórico de la Religión por Pinton" y el catecismo histórico del abate Fleury ${ }^{47}$. Sin embargo, en sus informes de 1790, ninguno de los maestros hacía referencia a esas obras. Si ese silencio en lo que respecta a los profesores particulares puede perfectamente interpretarse como testimonio de su ausencia en las lecturas de sus escuelas, no estamos tan seguros de que así fuera en la pública de la villa. La parquedad con la que el maestro titular D. Jacobo Violán aborda el informe en general y su mayor sujeción a los dictámenes reales, dado el carácter de realengo del ayuntamiento y su clara vocación reformista, pudieron hacer que se empleasen en la escuela pública. De todas maneras, dada la escasez de información ésta es una suposición que no cuenta con aval documental alguno hasta el momento.

Dentro del programa de reforma moral y religiosa que se pretendía transmitir en estas escuelas a las nuevas generaciones, no sólo tenía cabida el fomento de una religiosidad más sólida e intensa, sino también el desarrollo en la infancia de la "virtud y buenas costumbres", comportamientos estrechamente ligados a la propia ética cristiana. El 6 de mayo de 1790, el Consejo de Castilla incidía en la importancia de que los maestros, formasen la conducta de los niños "inspirándoles con su doctrina y exemplo buenas máximas morales y políticas" ${ }^{48}$. Todos los informes de los maestros ferrolanos elaborados ese mismo año, resaltaban la importancia que para todos ellos tenía fomentar entre los pequeños "la obediencia y sumisión", comenzando por el propio maestro, siguiendo por los padres y

45 A.M.F., Libro de consistorio $n^{\circ} 17$ (1790), fol. 241.

${ }^{46}$ A.M.F., Libro de consistorio $n^{\circ} 18$ (1790), fol. 169 vto.

${ }^{47}$ Pintón, J. (1754). Compendio histórico de la Religión desde la creación del mundo hasta el estado presente de la Iglesia por preguntas y respuestas para uso de la juventud, Madrid: Imprenta de Antonio Pérez de Soto; (1805-1807). Novísima Recopilación, Madrid, Lib. VIII, Tit. I, Ley III.

${ }^{48}$ A.M.F., Libro de consistorio $n^{\circ} 18$ (1790), fol. 41. 
terminando en las autoridades políticas y religiosas. De esta manera, en palabras de D. Antonio de Puente y Andrade, se buscaba "formar en sus discípulos unos buenos ciudadanos, así para el servicio de Dios como para el mayor bien del Estado"49. Esa sumisión a los mayores se manifestaba, por ejemplo, en el besamanos al maestro que debían hacer los niños de la escuela de Esteiro nada más llegar a clase, o el que exhortaba el de San Roque que hicieran los alumnos a sus padres una vez retornados a casa. También, se les inculcaba la práctica de besar las manos de todos los sacerdotes que se toparan por su camino. Para fomentar esta actitud de sumisión, los pequeños memorizaban los contenidos de la "cartilla de buena crianza", texto base de todas las escuelas de la villa. En la principal, lo hacían todos los jueves.

Además de los principios religiosos, políticos y de urbanidad, las escuelas de primeras letras introducían a los alumnos en el conocimiento de la lectura, la escritura y las reglas aritméticas más básicas ${ }^{50}$. Los niños comenzaban aprendiendo a leer, para pasar luego al aprendizaje de la escritura y, finalmente, a las operaciones de cálculo. Por ese motivo, la primera de esas enseñanzas era la que mayor difusión tenía entre las clases populares, mientras que la última la que menos, dado el abandono de un número considerable de alumnos antes de llegar a ese nivel. La lectura y la escritura eran pues dos mundos separados, debido en gran medida al importante coste económico que suponía el material de aprendizaje de la escritura, cuyos gastos duplicaban los de la lectura. Como es lógico suponer, la lengua empleada en la escuela, tanto por el maestro como por los alumnos, era el castellano. La Real Cédula de 23 de junio de 1768 así lo exigía para todos los centros de estudio a nivel español, si bien en el caso gallego ya se practicaba así en los siglos precedentes. El método habitual para aprender a leer en toda España era el deletreo, es decir, el conocimiento de cada una de las letras que componen el alfabeto, tanto en su forma minúscula como mayúscula, primero de manera lineal y después, una vez adquirida cierta pericia, de forma salteada. A continuación se comenzaba con el silabeo para, más tarde, lanzarse a la lectura sin interrupción. Se trataba de un método ciertamente árido que, además, se veía dilatado por la organización y distribución de las tareas escolares ${ }^{51}$. En consecuencia, los materiales básicos para la enseñanza de la lectura eran en Ferrol, como en tantos otros centros urbanos del territorio español, los tradicionales abecedarios y

\footnotetext{
49 A.M.F., Libro de consistorio $n^{\circ} 18$ (1790), fol. 237.

${ }^{50}$ Vid. GARCÍA HURTADO, M.R. (2004): "Reflexiones sobre algunos textos destinados a enseñar a leer y escribir en España entre 1700 y 1800”, Obradoiro de Historia Moderna, pp. 7.38.

${ }^{51}$ VIÑAO FrAGO, A. (1992). “Alfabetización, lectura y escritura...”, p. 52.
} 
silabarios. Siguiendo el "estilo del país", maestros como Tomás de la Carrera o Francisco Fernández, los iniciaban en los rudimentos de la lectura a través de manuscritos elaborados por ellos mismos. Fernández confeccionaba unas cartillas con "un silabario difuso compuesto por mi, en que se hallan casi todos ptongos, diptongos y triptongos de que se componen las dicciones de nuestra lengua". Una vez aprendido el silabario, el maestro les introducía en la lectura de "asuntos edificativos", como oraciones de la Iglesia, primero sobre la base de monosílabos y disílabos y después tomando frases más complejas ${ }^{52}$. Otros maestros, como Francisco Gamero o D. Antonio de Puente y Andrade, se decantaban por los abecedarios y silabarios impresos frente a los tradicionales manuscritos. Gamero, tenía colgado en lugar preferente de la escuela uno de ellos. D. Antonio, iba más allá, criticando duramente la práctica de sus colegas, al considerar que la lectura de manuscritos generaba irreparables vicios de lectura en los pequeños ${ }^{53}$.

Adquirido ya un nivel aceptable, ya fuera a través de los silabarios impresos o de los manuscritos, los alumnos pasaban seguidamente al catón, para adquirir ligereza en el silabeado e incluso, los más avanzados, alcanzar la perfección de la lectura con todos sus matices, respetando los signos de puntuación y acentos. A partir de entonces, los que continuaban con los estudios, podían acceder ya a la lectura de obras de mayor enjundia. Varias disposiciones regias a lo largo del siglo, defendían la ausencia en las lecturas escolares de libros de ficción, es decir, de "novelas, romances, comedias o historias profanas", por el "considerable daño para la puerilidad". Se recomendaban las obras de fondo religioso, como los propios catecismos históricos de Fleury o Pintón, o el "Camino de la Sabiduría" de Luis Vives, "cuya obra es la más apropósito para instruir a los niños de tierna edad en todas las obligaciones que constituyen un cristiano verdadero y un buen ciudadano" 54 . A ellos se añadía, a partir de 1771, la posibilidad de elegir "algún compendio de la historia de la Nación", primer tímido intento de introducir el estudio de la Historia en el currículum escolar ${ }^{55}$.

El aprendizaje de la escritura se iniciaba solamente cuando el alumno dominaba de una manera aceptable la lectura. Era un proceso mucho más caro que el anterior;

52 A.M.F., Libro de consistorio $n^{\circ} 18$ (1790), fols. 229-229 vto.

53 A.M.F., Libro de consistorio $n^{\circ} 18$ (1790), fol. 236.

${ }^{54}$ Ver, Luzuriaga, L. (1916). Documentos para la historia escolar de España, Madrid: JaeCentro de Estudios Históricos.

55 Así se afirmaba en la Real Provisión de Felipe V de 20 de diciembre de 1743, opinión ratificada por Carlos III en 1763. Novísima Recopilación, Lib. VIII, Tit. I, Ley IV; RUIZ BERRIO, J. (1998). "La educación del pueblo español...”, pp. 172-175. 
los gastos se duplicaban, dado el costoso material que había que manejar -plumas, tinteros, tinta, papel, etc.-. Además, se trataba de un sistema de enseñanza ciertamente lento, puesto que el método caligráfico se asemejaba más al dibujo que a una escritura simplificada ${ }^{56}$. Escribir era todo un arte, como defendía el conservador Francisco Javier de Santiago Palomares en su influyente tratado. De hecho, todas las escuelas ferrolanas aplicaban en 1790 su método, utilizando bien la obra original, o la posterior actualización llevada adelante por Esteban Jiménez $^{57}$. Dada esa coincidencia a la hora de elegir la base teórica de las enseñanzas de escritura, el método empleado en los distintos centros era muy similar. Palomares concebía la caligrafía como una disciplina del cuerpo y un arte del trazo, según la cual, era imprescindible para el aprendizaje la imitación y la copia. Los primeros pasos del alumno consistían en aprender a tomar la pluma con corrección, poniendo el brazo en la postura adecuada, tal y como mostraban los grabados de Palomares. Una vez adquirida la posición correcta, comenzaban a practicar los trazos de la escritura con una pluma sin tinta, siguiendo con ella el contorno de unas letras escritas o impresas en papel. El maestro de Esteiro, Francisco Gamero, introducía en esta fase del aprendizaje la novedad de emplear unas "plantas señaladas con plomo, las que los niños rellenan con la tinta y pluma, de lo que se consigue que a poco tiempo toman con facilidad el nuevo método" ${ }^{\text {. }}$. A partir de entonces, comenzaban todos con la escritura a través del método de copia y repetición de letras mayúsculas y minúsculas, a la vez que se les enseñaban "los tres trazos o rayas que da la pluma en diferente grueso", procurando que los pequeños aprendiesen a formar las letras "con uniformidad y semejanza".

El único maestro que se separaba un tanto de esta tónica general era D. Antonio de Puente y Andrade, que junto los tratados de Palomares y Jiménez, empleaba las reglas de escritura de los padres escolapios, desarrolladas en la obra del padre Felipe Scío de San Miguel $^{59}$. Esta orden abogaba por una mayor sencillez y claridad de la escritura, a fin de satisfacer la creciente demanda social de este saber. De hecho, durante las últimas décadas del siglo XVIII se desarrolló en España una

${ }^{56}$ VIÑAO FRAGO, A. (1992). “Alfabetización, lectura y escritura...”, p. 52.

${ }^{57}$ Santiago Palomares, F.J. (1776). Arte nueva de escribir: inventada por Pedro Díaz Morante e ilustrada por D. Francisco Xavier de Santiago Palomares, Madrid: Imprenta de D. Antonio de Sancha; JimÉneZ, E. (1789). Arte de escribir: siguiendo el método y buen gusto de D. Francisco Xavier de Santiago Palomares, Madrid: Imprenta de Benito Cano.

58 A.M.F., Libro de consistorio $n^{\circ} 18$ (1790), fol. 167 vto.

${ }^{59}$ Scio De SAN Miguel, F. (1780). Método uniforme para las escuelas de cartilla, deletrear, leer, escribir, aritmética, gramáticas castellana y ejercicio de doctrina cristiana, como se practica por los padres de las Escuelas Pías, Madrid: Imprenta de Pedro Marín. 
intensa polémica entre los defensores del método tradicional, los llamados "palomaristas", y aquellos que se decantaban por la simplificación del trazo y la enseñanza sin muestras, agrupados en torno a la obra de José Anduaga y Garimberti y el "movimiento de San Ildefonso" ${ }^{\circ 0}$. La victoria de estos últimos se produjo en 1793, año en el que el tratado de Anduaga quedó establecido como manual oficial para la caligrafía. En 1802, se sustituía esa obra por la del calígrafo palentino Torcuato Torío de la Riva, publicada en 1798, manteniéndose como principal libro de texto durante el resto del reinado de Carlos IV. El libro junto a la historia y teoría de la escritura, incluía nociones de gramática, aritmética, urbanidad y didáctica ${ }^{61}$. A la par que se producía un proceso de simplificación de la escritura en la enseñanza, las medidas legislativas de tiempos de Carlos III supusieron también la normalización y fonetización ortográfica, sobre la base de las publicaciones de la Real Academia Española. Las primeras ediciones de las reglas de Ortografía y Gramática de la Academia datan de 1741 y 1771 respectivamente, siendo objeto de sucesivas reimpresiones y modificaciones durante la segunda mitad del XVIII. A partir de 1780, todas las escuelas españolas debían enseñar la lengua castellana a través de la gramática de la Academia, previniendo que no se admitiría a ninguno a estudiar latinidad, sin que constase su adecuada instrucción en la gramática española ${ }^{62}$.

El último paso en el aprendizaje eran los estudios de aritmética, cuya pobreza de objetivos resultaba también evidente, limitándose a introducir en el niño las reglas matemáticas básicas para su empleo en la vida cotidiana. El desinterés por las operaciones matemáticas para la enseñanza en primeras letras queda perfectamente reflejado en los exámenes de los maestros, en los cuales el principal requisito para obtener el aprobado y el consiguiente título era la pericia caligráfica. En los expedientes de exámenes conservados en Archivo Histórico Nacional, puede contemplarse como la prueba de escritura ocupaba un número considerable de páginas, mientras que las operaciones matemáticas -sumas, restas, multiplicaciones, divisiones y raíces cuadradas- solían aparecen en los estrechos márgenes de alguna hoja. El maestro de la calle real, Puente y Andrade, enseñaba a sus pupilos según su propio testimonio "las quatro o cinco reglas de enteros, las de quebrados, las de enteros con quebrados, las de denominados, las de tres simples y

60 Anduaga y Garimendi, J. (1781). Arte de escribir por reglas y sin muestras, Madrid: Imprenta Real.

${ }^{61}$ TORÍ́ De LA Riva Y HeRrero, T. (1798). Arte de escribir por reglas y con muestras, Madrid: Imprenta de la viuda de Joaquín Ibarra.

${ }^{62}$ Aguilar Piñal, A. (1987). “La política docente...”, p. 445. 
compuestos, las de compañía con tiempo, las de testamento y otras varias y útiles. Haciendo que primero formen bien los números, los lean y sepan la tabla de quentas". Francisco Fernández utilizaba "ejemplos mercantiles y reducciones de pesos y medidas", con el objetivo de enseñar "no sólo el algoritmo de enteros sino el de quebrados, números complexos, extracción de raíces, reglas de tres simples y compuestas, compañías, testamentos, repartimientos y aligaciones". Por su parte, Tomás de la Carrera obligaba a sus alumnos a aprender la tabla "de seguido y salteada", además de manejar las cinco reglas simples ${ }^{63}$.

\section{LA EDUCACIÓN FEMENINA}

Si para la enseñanza elemental masculina contamos con unas fuentes de información más que aceptables, no ocurre lo mismo en lo que respecta a la femenina. Los testimonios sobre la existencia de escuelas de educación para niñas en Ferrol durante la segunda mitad del siglo XVIII son contados y cuando aparecen están más relacionados con la represión de determinadas infracciones cometidas por las maestras que con una verdadera preocupación por sus planes de estudios. Lo que es indudable es que, en general para toda España, los niveles de escolarización de las niñas eran significativamente más bajos que los de los niños. Sirva como muestra los datos del Censo de 1797 , en el que se contabilizaban un total de 8.704 varones cursando los primeros estudios, frente a solamente 2.303 mujeres $^{64}$. A esa significativa inferioridad en cuanto a escolarización, hay que añadir una menor ambición en los objetivos de la enseñanza en el campo femenino. Estos pobres objetivos tenían también su reflejo en las mínimas exigencias que se requerían para ejercer las docencia femenina, frente a las mayores que debían acreditar los maestros de primeras letras: a las mujeres que quisieran convertirse en maestras de las "escuelas de enseñanza", solamente se les requería un informe de vida y costumbres y la prueba de haber superado la doctrina cristiana. Por ese motivo, no resultaba extraño encontrarse aún a finales del siglo XVIII con maestras que no supieran escribir.

En las "escuelas de enseñanza", llamadas así para diferenciarlas de las de "primeras letras" masculinas, las profesoras, atendiendo a lo estipulado por la Real Cédula de 11 de mayo de 1783, debían ejercitar a las pequeñas en doctrina cristiana así como en las labores "propias de su sexo". La lectura podía tener cabida, aunque no en todas las escuelas, mientras que la escritura era descartada absolutamente. En consecuencia, el escaso número de niñas que lograba acceder al sistema educativo,

\footnotetext{
${ }^{63}$ A.M.F., Libro de consistorio $n^{\circ} 18$ (1790), fols. 233 vto., 236 vto. y 241 vto.

${ }^{64}$ VIÑAO FrAGO, A. (1992). “Alfabetización, lectura y escritura...”, p. 51.
} 
alcanzaba, en el mejor de los casos, una pobre semialfabetización -alfabetización lectora- limitada a un catecismo memorizado. $\mathrm{Y}$ es que los peligros morales que podía conllevar la enseñanza de la lectura y la escritura a la mujer era cuestión muy difundida en un significativo número de tratados de la época. La excesiva cultura, en opinión de muchos, lo único que podía hacer era sembrar confusión en las simples mentes femeninas, alejándolas de sus principales cometidos en la vida matrimonial. Por ese motivo, en una conocida obra de finales del siglo XVIII se señalaba tajantemente que "un marido debe desear que su muger se instruya únicamente en agradarle, y en cumplir todas aquellas tiernas obligaciones que la impusieron la naturaleza, la religión y el Estado"65.

Para el caso específico del Ferrol del XVIII, conocemos la existencia de un número indeterminado de escuelas para niñas que podían llegar "asta el número de diez, ocultas y públicas", según narraba de modo poco concreto una aspirante a obtener la titulación de maestra en la década de los ochenta. Sin embargo, y a diferencia de las escuelas de primeras letras, no contamos para los centros femeninos con una estadística oficial. El menor control ejercido por el municipio ferrolano a estos centros responde al ya referido discreto interés que suscitaba la educación de las niñas en la época. Las únicas medidas de cierto fuste tomadas por el regimiento departamental durante todo el XVIII con respecto a la enseñanza femenina estuvieron relacionadas con la administración de Álvarez Caballero. En 1784 , el alcalde mayor intentó poner un cierto orden en el desorganizado mundo de la enseñanza primaria femenina, procurando hacer cumplir en las escuelas de niñas la legislación vigente. En consecuencia, aquellas mujeres que quisieran ejercer la profesión docente, debían pedir al municipio la autorización de rigor. En la documentación emanada de la ordenanza municipal, ha quedado constancia de dos de ellas: $\mathrm{D}^{\mathrm{a}}$. Juliana Maté y $\mathrm{D}^{\mathrm{a}}$. Agustina Martínez de la Muria. La primera era hija de un contador de la Armada por aquellas fechas ya fallecido, y tenía abierta, desde hacía tres años, una escuela en el barrio de Esteiro junto a la puerta de Caranza. En ella, enseñaba a sus educandas a "leer y escribir, calzetar y coser". Su intención era obtener del concejo la correspondiente autorización para abrir una escuela en el barrio de Ferrol Viejo "por haver quedado huérfana en compañía de su Madre (por no tener más de terceras de viudedad y ser su ánimo permanecer en su compañía en el estado de soltera)". Por su parte, $\mathrm{D}^{\mathrm{a}}$. Agustina, llevaba quince años regentando una escuela en la calle de San Carlos, en pleno barrio de Esteiro. Estaba casada con un asturiano natural del puerto de Navia y aseguraba, como argumento de peso para obtener la autorización municipal, tener papeles de nobleza.

65 (1792). Consideraciones politicas sobre la conducta que debe observarse entre marido y mujer. Parte primera, Madrid: Ramón Ruiz, p. 124. 
Esa fue la única ocasión en la que existió un verdadero interés por reglamentar la educación femenina en el Ferrol dieciochesco y los resultados no dejaron de ser bastante discretos, ya que las escuelas ilegales de niñas siguieron existiendo, sin que el poder político pudiese atajar el problema definitivamente. Tras este fracaso de Álvarez Caballero, sus sucesores se limitaron casi exclusivamente a abortar ciertas prácticas fraudulentas de algunas de estas maestras, que eran consideradas extremadamente peligrosas para el correcto desarrollo de la educación en la real villa. $\mathrm{Y}$ es que varias mujeres que regentaban escuelas de niñas, desoyendo las ordenanzas municipales y leyes del reino, admitían sin miramientos en sus centros educativos a estudiantes varones. Ya hemos visto como la mezcla de sexos en la escuela era considerada perniciosa para la correcta educación sobre todo de los varones, pero quizás mucho más grave era que precisamente éstos estuvieran dirigidos en sus primeros pasos educativos por una mujer, habida cuenta de la subestimación que había de sus capacidades intelectuales y de su apego a los vicios mundanos. Pero, como en otros muchos aspectos de la vida ciudadana, la incapacidad humana y económica del concejo para hacer frente a la multitud de problemas que le acosaban, hizo que estas medidas puntuales no lograsen terminar con esta práctica. En diciembre de 1810, el procurador general de la villa, D. Ramón Vázquez Carreño contabilizaba nada menos que veintitrés escuelas ilegales $^{66}$. En se mismo año, solamente existía un establecimiento de educación primario para niñas reconocido: el situado en el número treinta y nueve de la calle Dolores y regentado por $\mathrm{D}^{\mathrm{a}}$. Claudia Ribera, esposa del maestro titular de la villa y que, cumpliendo las leyes, solamente admitía bajo su techo a niñas. Este mediocre panorama pervivirá bastante más allá del final del Antiguo Régimen.

${ }^{66}$ A.M.F., Instrucción pública, Caja 775. 